\title{
Population pharmacokinetics modeling of oxcarbazepine to characterize drug interactions in Chinese children with epilepsy
}

\author{
Yang WANG, Hua-nian ZHANG ${ }^{1, *}$, Chang-he NIU, Ping GAO, Yu-jun CHEN, Jing PENG, Mao-chang LIU, Hua XU \\ Department of Pharmacy, Wuhan Children's Hospital, 100th-Hong Kong Road, Wuhan 430016, China
}

\begin{abstract}
Aim: To develop a population pharmacokinetics model of oxcarbazepine in Chinese pediatric patients with epilepsy, and to study the interactions between oxcarbazepine and other antiepileptic drugs (AEDs).

Methods: A total of 688 patients with epilepsy aged 2 months to 18 years were divided into model $(n=573)$ and valid $(n=115)$ groups. Serum concentrations of the main active metabolite of oxcarbazepine, 10-hydroxycarbazepine (MHD), were determined 0.5-48 h after the last dosage. A population pharmacokinetics (PPK) model was constructed using NLME software. This model was internally evaluated using Bootstrapping and goodness-of-fit plots inspection. The data of the valid group were used to calculate the mean prediction error (MPE), mean absolute prediction error (MAE), mean squared prediction error (MSE) and the $95 \%$ confidence intervals $(95 \% \mathrm{Cl})$ to externally evaluate the model.

Results: The population values of pharmacokinetic parameters estimated in the final model were as follows: $K_{a}=0.83 \mathrm{~h}^{-1}, V_{\mathrm{d}}=0.67$ $\mathrm{L} / \mathrm{kg}$, and $\mathrm{CL}=0.035 \mathrm{~L} \cdot \mathrm{kg}^{-1} \cdot \mathrm{h}^{-1}$. The enzyme-inducing AEDs (carbamazepine, phenytoin, phenobarbital) and newer generation AEDs (levetiracetam, lamotrigine, topiramate) increased the weight-normalized CL value of MHD by $17.4 \%$ and $10.5 \%$, respectively, whereas the enzyme-inhibiting AED valproic acid decreased it by $3 \%$. No significant association was found between the CL value of MHD and the other covariates. For the final model, the evaluation results $(95 \% \mathrm{Cl})$ were $\mathrm{MPE}=0.01(-0.07-0.10) \mathrm{mg} / \mathrm{L}, \mathrm{MAE}=0.46(0.40-0.51) \mathrm{mg} / \mathrm{L}$, MSE $=0.39(0.27-0.51)(\mathrm{mg} / \mathrm{L})^{2}$.

Conclusion: A PPK model of OXC in Chinese pediatric patients with epilepsy is established. The enzyme-inducing AEDs and some newer generation AEDs (lamotrigine, topiramate) could slightly increase the metabolism of MHD.
\end{abstract}

Keywords: epilepsy; oxcarbazepine; 10-hydroxycarbazepine; population pharmacokinetics; drug interaction; CYP450; pediatric patients; Chinese children

Acta Pharmacologica Sinica (2014) 35: 1342-1350; doi: 10.1038/aps.2014.76; published online 15 Sep 2014

\section{Introduction}

Oxcarbazepine (10,11-dihydro-10-oxo-5H-dibenz[b,f]azepine5 -carboxamide; OXC) is a newer antiepileptic drug that has been widely used in many countries for the treatment of partial seizures (including the seizure subtypes of simple, complex, and partial seizures evolving to secondarily generalized seizures) as both a monotherapy and an adjunctive therapy in children and adults with epilepsy ${ }^{[1]}$. The antiepileptic properties of OXC are possibly mediated through its effects on neuronal flux and specifically, by blocking the voltage dependent sodium channel ${ }^{[2]}$.

OXC, the 10-keto derivative of carbamazepine (CBZ), has a

\footnotetext{
* To whom correspondence should be addressed.

E-mail cattop3211@sohu.com

Received 2014-03-04 Accepted 2014-07-04
}

similar clinical efficacy as CBZ, but with fewer adverse drug reactions and better tolerability ${ }^{[3,4]}$. The oral bioavailability of OXC is above $95 \%$. Following oral administration, OXC is rapidly reduced by cytosolic arylketone reductases in the liver to 10-hydroxycarbazepine (MHD), which is responsible for the pharmacological effects of the drug. MHD is eliminated through conjugation with glucuronic acid and subsequently excreted in the urine ${ }^{[4-6]}$. In humans, the formation of MHD is stereoselective, with the two enantiomeres formed in a ratio of $80 \%$ (S-MHD) to $20 \%(R-M H D)^{[4-8]}$. It has been demonstrated that the two enantiomers have similar antiepileptic efficacy and tolerability ${ }^{[9]}$. Usually, the pharmacokinetics and disposition of the racemate were investigated ${ }^{[4]}$. A number of studies ${ }^{[2,3,10]}$ have reported a linear relationship between OXC doses and MHD concentrations in patients and healthy volunteers, and MHD accounted for the majority of the radio- 
active species circulating in plasma, with only low levels of unchanged OXC; therefore, OXC acts as a prodrug of $\mathrm{MHD}^{[4]}$. MHD was typically the target of the pharmacokinetic or pharmacodynamic investigations of OXC.

Several antiepileptic drugs, such as CBZ, phenytoin (PHT) and phenobarbital (PB) are metabolized by the cytochrome P450 (CYP450) oxidative processes. Unlike these drugs, OXC primarily undergoes reductive biotransformation. Therefore, OXC has a low propensity of autoinduction and enzyme induction. Because of this distinct difference, OXC has a small potential for drug-drug interactions with other antiepileptic drugs (AEDs) ${ }^{[2]}$. Thus, it has been suggested that there was no relationship between concomitant administration with other AEDs and the apparent clearance of $\mathrm{MHD}^{[10]}$. However, some studies have reported a higher apparent clearance and lower MHD concentrations when OXC was coadministered with enzyme-inducing medications ${ }^{[1-3,11]}$. Furthermore, it has been demonstrated that OXC was a weak inhibitor of CYP2C19 and may increase the serum concentration of $\mathrm{PHT}$ and $\mathrm{PB}^{[2,5,12]}$. Additionally, it is a weak inducer of uridine glucuronyl transferases (UGT1A4), as demonstrated by a $29 \%$ decrease in the serum concentration of lamotrigine $(\mathrm{LTG})^{[13,14]}$. At present, these ideas remain controversial and the mechanism has not been fully elucidated.

There is limited pharmacokinetic information available regarding Chinese pediatric patients with epilepsy treated with OXC. Therefore, the aims of the present study were to develop a population pharmacokinetics (PPK) model of OXC in Chinese children with epilepsy and to investigate the pharmacokinetic characteristics of OXC including the influence of other antiepileptic drugs on the pharmacokinetic parameters of OXC in these subpopulations, which will facilitate individualized dosage regimens.

\section{Materials and methods}

\section{Patients}

Children with epilepsy were recruited by pediatricians in the Department of Neurology at Wuhan Children's Hospital from June 2011 to February 2013. The enrollment criteria were as follows: (1) the children were diagnosed with epilepsy based on the criteria of International League Against Epilepsy (ILAE); (2) the children had good compliance in following medical orders or other requirements of this study; (3) the patients had normal liver and kidney function during drug treatment; and (4) the patients did not receive special treatments that could impact drug elimination, such as dialysis and diuresis. The exclusion criteria were as follows: (1) missing some critical research information; (2) blood samples were taken at the wrong time or without meeting the requirements; and (3) other factors that the clinicians or researchers considered to be unsuitable for this study. In total, 688 cases of pediatric patients with epilepsy aged 2 months to 18 years were collected in this study. The patients were from all over the country and were taking OXC alone or concomitantly with other AEDs. For each patient, the gender, age, weight and coadministered medications were recorded. The data were randomly divided into 2 groups, the PPK model group $(n=573)$ and the PPK valid group $(n=115)$, using SPSS (Statistical Package for the Social Sciences) 13.0 software. The demographic and clinical characteristics of the pediatric patients are summarized in Table 1. The protocol for this study was approved by the Medical Ethics Committee of Wuhan Children's Hospital, and written informed consent was obtained from their guardians (ie, their parents).

\section{OXC administration and sample collection}

OXC tablets were administered orally in daily doses of 10-30

Table 1. Pertinent patient characteristics of ALL study population.

\begin{tabular}{|c|c|c|}
\hline Item & PPK model group & PPK valid group \\
\hline \multicolumn{3}{|l|}{ Patient data } \\
\hline Gender (male:female) & $365: 208$ & $72: 43$ \\
\hline Mean age (months) (range) & $88.46 \pm 43.17(2-216)$ & $85.35 \pm 41.83(7-192)$ \\
\hline Mean weight (kg) (range) & $27.64 \pm 12.93(4-90)$ & $25.49 \pm 11.25(8-73)$ \\
\hline \multicolumn{3}{|l|}{ Sample data } \\
\hline Total № of treatment courses & 1027 & 222 \\
\hline Total № of concentration-time points & 1031 & 223 \\
\hline № of observations per subject & $1-10$ & $1-9$ \\
\hline Mean dose $\left(\mathrm{mg} \cdot \mathrm{kg}^{-1} \cdot \mathrm{d}^{-1}\right)$ (range) & $21.89 \pm 8.48(4.55-69.23)$ & $22.43 \pm 8.53(4.84-65.00)$ \\
\hline \multicolumn{3}{|l|}{ Comedication information } \\
\hline OXC+NEWAEDs (LEV/LTG/TPM) & $49(12 / 31 / 6)$ & $10(6 / 4 / 0)$ \\
\hline OXC+VPA & 36 & 6 \\
\hline OXC+EIAEDs (CBZ/PB) & $16(9 / 7)$ & $8(6 / 2)$ \\
\hline
\end{tabular}


$\mathrm{mg} / \mathrm{kg}$, qd, bid, or tid. The OXC dose regimen could be adjusted in cases of inadequate seizure control or side effects, as shown in Table 1. Patients fasted for one hour before and after drug administration. Blood samples of approximately $3 \mathrm{~mL}$ were taken, and the time between dosing and sampling was recorded. Most of the samples were taken at the end of the dosing interval, and the concentrations had reached a steady state $\left(C_{\mathrm{ss}}\right)$. The blood samples were centrifuged, and the separated serum was preserved at $-20^{\circ} \mathrm{C}$ for less than a week before analysis.

\section{Serum MHD assay}

Serum samples were extracted using a solid phase column extractor (ODS $\mathrm{C}_{18}, 200 \mathrm{mg} / 3 \mathrm{~mL}$ ), and MHD was detected using HPLC (Agilent 1260). The separation was performed on a Venusil $\mathrm{C}_{18}$ column $(4.6 \times 250 \mathrm{~mm}, 10 \mu \mathrm{m})$ with a mobile phase of methanol:water (45:55) at a flow rate of $0.8 \mathrm{~mL} / \mathrm{min}$. The eluent was monitored at $230 \mathrm{~nm}$. This method had good specificity between analyte and matrix. A good linearity was observed over the concentration range of $2.49-39.82 \mathrm{mg} / \mathrm{L}$ with a correlation coefficient of 0.9998 . The limit of detection (LOD) was $0.4 \mathrm{mg} / \mathrm{L}$; the mean recoveries of $\mathrm{MHD}$ in the QC samples at concentrations of 2.49, 19.91, and $39.82 \mathrm{mg} / \mathrm{L}$ were between $97.2 \%-101.2 \%$, and intra- and inter-day precision RSD were all less than $10 \%$.

\section{Population PK model development}

\section{Software and hardware}

The population PK analysis was performed using a non-linear mixed-effect model approach implemented using the Phoenix ${ }^{\circledR}$ NLME computer program (version 1.2, Pharsight Corporation, USA) running on a Dell precision ${ }^{\circledR}$ desktop computer (version T5500, Dell Inc, China) with the Windows 7 operating system.

\section{Structural model}

Based on previous research ${ }^{[2,5,15,16]}$, we first proposed the following assumptions: OXC was completely absorbed (the bioavailability was fixed at 1.0) and metabolized to the same amount of MHD in the mass unit. The first-order conditional estimation method with the $\eta-\varepsilon$ interaction option (FOCE L-B) was used throughout the model building process. Based on previous modeling performed on other studies ${ }^{[2,3]}$ and exploratory graphical analysis, one- and two-compartment structural kinetic models with first-order elimination were evaluated. The best structural model was chosen based on an examination of the objective function value (OBJ, equal to the $-2 \log$ likelihood value of the data) and the visual inspection of standard goodness-of-fit plots, including the individual fits. Finally, a one-compartment, open kinetic model with firstorder absorption and elimination best described the data.

\section{Statistical model}

The exponential model was used to describe the inter-individual variability of the PK parameters as shown in Equation 1:

$$
P_{\mathrm{i}}=\theta \cdot \operatorname{Exp}\left(\eta_{\mathrm{i}}\right)
$$

Where $P_{\mathrm{i}}$ is the pharmacokinetic parameter for the ith individual, $\theta$ is the population typical value of the according parameter, and $\eta_{i}$ is a random variable for the ith individual following a Gaussian distribution with a mean of 0 and a variance of $\omega^{2}$.

The intra-individual variability (residual error) was evaluated using an addition model according to Equation 2:

$$
C_{\text {obs }}=C_{\text {pred }}+\varepsilon
$$

Where $C_{\text {obs }}$ is the observed serum MHD concentration, $C_{\text {pred }}$ is the corresponding model predicted concentration, and $\varepsilon$ is assumed to follow a Gaussian distribution with a mean of 0 and a variance of $\sigma^{2}$.

\section{Covariate analysis}

In the first step, the basic model was parameterized for apparent clearance (CL), the apparent volumes of distribution of the central compartment $\left(V_{\mathrm{d}}\right)$, and the absorption rate constant $\left(K_{\mathrm{a}}\right)$ without covariates added. In the second step, demographic variables (this study included sex, age, weight, and body surface area) and comedication (CO) were tested as potential covariates for the PK parameters. Based on previous studies ${ }^{[2,3]}$, we considered $\mathrm{CO}$ to be a potential covariate explaining the kinetic differences. $\mathrm{CO}$ was included as a categorical variable [0 for OXC monotherapy; 1 for comedication with newer AEDs (NEWAEDs) such as levetiracetam (LEV), LTG and topiramate (TPM); 2 for comedication with enzymeinhibiting AEDs such as valproic acid (VPA); and 3 for comedication with enzyme-inducing AEDs (EIAEDs) such as CBZ and $\mathrm{PB}$. Categorical covariates (sex and $\mathrm{CO}$ ) were incorporated using indicator variables with an exponential function. The influences of continuous covariates, such as age, weight, and body surface area were included in the model using a power function after normalization to the median value. A visual covariate screening procedure was performed before modeling. For visual screening, scatterplots for continuous variables and boxplots for discrete variables were used. Variables showing a potential relationship with a certain PK parameter in the screening procedure were included in the model to be selected as important covariates. In the third step, when the important covariates were selected, covariates were chosen using stepwise forward selection-backward elimination with the likelihood ratio test. Because the OBJ follows a chi-square distribution, a covariate was considered significant when the addition of the covariate resulted in a decrease in the OBJ $(\triangle \mathrm{OBJ})$ of greater than $3.84(P<0.05 ; \mathrm{df}=1)$, and the elimination of the covariate resulted in an increase in the OBJ $(\triangle \mathrm{OBJ})$ of greater than $6.63(P<0.01 ; \mathrm{df}=1)$. After a full covariate regressive model was established, the analysis procedure was continued until only significant covariates remained in the model. Then, the final model was established.

\section{Model evaluation}

The performance of the final model was internally evaluated both numerically and visually using bootstrapping and diagnostic plots, including goodness-of-fit and distribution of residuals. Using the final model, 1000 bootstrap-resampled 
data sets from the original model group data set were sequentially estimated. The median and $95 \%$ confidence intervals (95\% CIs) (2.5th percentile and 97.5th percentile) of the parameters obtained from this step were compared with the final parameter estimates. The serum concentrations of the valid group patients were predicted by the final model, and the mean predicted error (MPE), mean absolute prediction error (MAE), mean squared prediction error (MSE) and the 95\% CIs were calculated to externally evaluate the final model according to Equations 3-5.

$$
\begin{aligned}
& M P E=\frac{1}{n} \sum_{i=1}^{n}\left(C_{\mathrm{obsi}_{\mathrm{i}}}-C_{\text {pred }_{\mathrm{i}}}\right) \\
& M A E=\frac{1}{n} \sum_{i=1}^{n}\left|C_{\mathrm{obsi}_{\mathrm{i}}}-C_{\text {predi }_{\mathrm{i}}}\right| \\
& M S E=\frac{1}{n} \sum_{i=1}^{n}\left(C_{\mathrm{obsi}_{\mathrm{i}}}-C_{\text {predi }_{\mathrm{i}}}\right)^{2}
\end{aligned}
$$

Where $C_{\text {obsi }}$ is the observed serum MHD concentration of ith patient, and $C_{\text {predi }}$ is the final model predicted concentration.

\section{Statistical analyses}

Descriptive statistics were expressed as the arithmetic mean $\pm S D$ unless otherwise specified. The statistical analysis was carried out using SPSS Statistics for Windows software (version 13.0) by Student's $t$-test and one-way analysis of variance followed by post hoc test. $P<0.05$ was considered significant.

\section{Results}

\section{Patient characteristics}

A total of 1254 sampling points obtained from 1249 courses of treatment in 688 patients aged 2 months to 18 years ( 1 to 14 years accounted for $93.5 \%$ ) were included in the total dataset. The characteristics of the studied population are summarized in Table 1. The intervals between the last dosage time and sampling time were distributed over $0.5-48 \mathrm{~h}$ (Figure 1).

\section{PPK modeling}

The basic model was as follows:

$$
\begin{aligned}
& K_{\mathrm{a}}\left(\mathrm{h}^{-1}\right)=\theta_{\text {Ka }} \cdot \operatorname{Exp}\left(\eta_{\text {Ka }}\right) ; \\
& V_{\mathrm{d}}(\mathrm{L} / \mathrm{kg})=\theta_{V \mathrm{Vd}} \cdot \operatorname{Exp}\left(\eta_{\mathrm{Vd}}\right) ; \\
& \mathrm{CL}\left(\mathrm{L}^{\mathrm{kg}} \mathrm{kg}^{-1} \cdot \mathrm{h}^{-1}\right)=\theta_{\mathrm{CL}} \cdot \operatorname{Exp}\left(\eta_{\mathrm{CL}}\right)
\end{aligned}
$$

A summary of the full model development process is shown in Table 2 in decreasing order of the OBJ (model improvement). In the backward step, only comedication with other AEDs
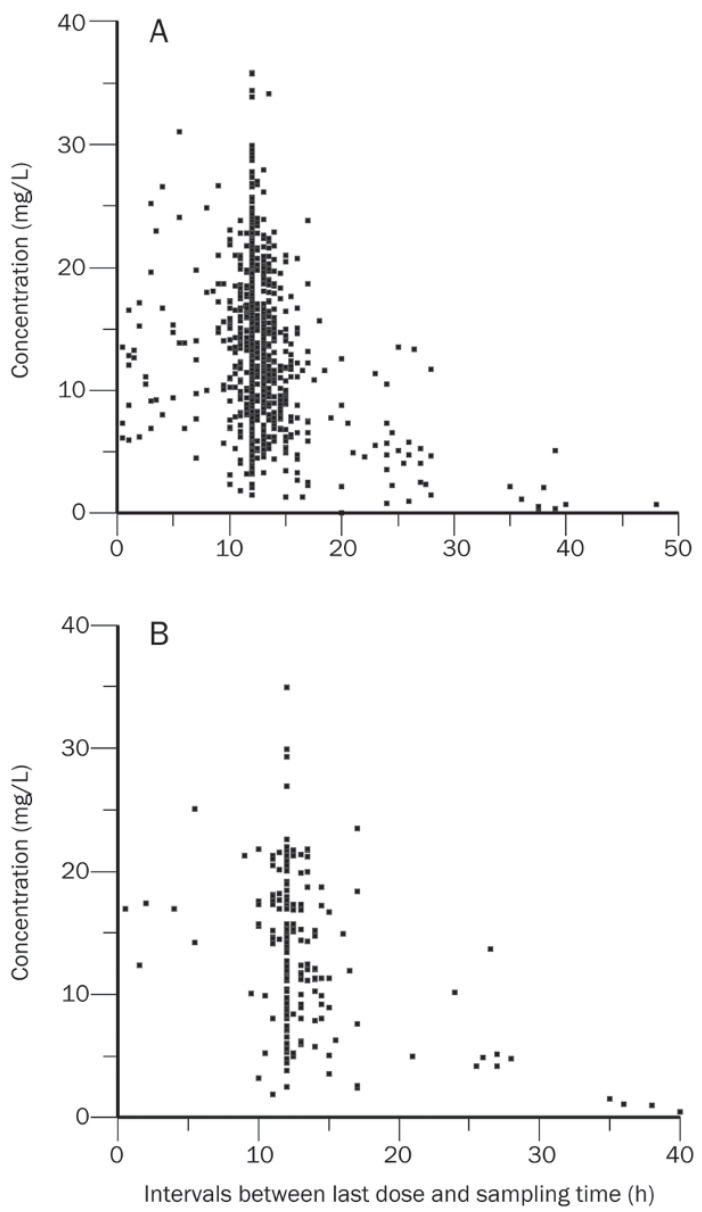

Figure 1. Scattergram of concentrations and intervals between the last dosage time and the sampling time of patients. (A) The PPK model group. (B) The PPK validation group.

(CO) was included as a statistically meaningful covariate for CL. No significant association was found between the other covariates and the apparent clearance of MHD.

The final model was as follows:

$$
\begin{aligned}
& K_{\mathrm{a}}\left(\mathrm{h}^{-1}\right)=\theta_{K a} \cdot \operatorname{Exp}\left(\eta_{K \mathrm{a}}\right) ; \\
& V_{\mathrm{d}}(\mathrm{L} / \mathrm{kg})=\theta_{V \mathrm{~d}} \cdot \operatorname{Exp}\left(\eta_{V \mathrm{~d}}\right) ;
\end{aligned}
$$

\begin{tabular}{|c|c|c|c|c|c|}
\hline Step & Model structure & OBJ & $\Delta \mathrm{OBJ}$ & $P$-value & Comments \\
\hline 0 & $C L=\theta_{C L} \cdot \operatorname{Exp}\left(\eta_{C L}\right)$ & 6025.9 & & & Basic model \\
\hline 1 & $C L=\theta_{C L} \cdot \operatorname{Exp}\left(\theta_{\text {weight }}\right) \cdot \operatorname{Exp}\left(\eta_{C L}\right)$ & 6000.8 & -25.1 & $<0.01$ & \\
\hline 2 & $\mathrm{CL}=\theta_{\mathrm{CL}} \cdot \operatorname{Exp}\left(\theta_{\mathrm{CO}}\right) \cdot \operatorname{Exp}\left(n_{\mathrm{CL}}\right)$ & 5928.6 & -97.3 & $<0.01$ & \\
\hline 3 & $C L=\theta_{C L} \cdot \operatorname{Exp}\left(\theta_{c o}\right) \cdot \operatorname{Exp}\left(\theta_{\text {weight }}\right) \cdot \operatorname{Exp}\left(n_{C L}\right)$ & 5923.4 & -5.2 & $<0.05$ & Full model \\
\hline
\end{tabular}

$\mathrm{CL}\left(\mathrm{L} \cdot \mathrm{kg}^{-1} \cdot \mathrm{h}^{-1}\right)=\theta_{\mathrm{CL}} \cdot \operatorname{Exp}\left(\theta_{\mathrm{NEWAED}}\right) \operatorname{Exp}\left(\theta_{\mathrm{VPA}}\right) \cdot \operatorname{Exp}\left(\theta_{\mathrm{EIAEDS}}\right) \cdot \operatorname{Exp}\left(\eta_{\mathrm{CL}}\right)$ Where $\theta_{\mathrm{NEWAEDs}}, \theta_{\mathrm{VPA}}$, and $\theta_{\mathrm{EIAEDS}}$ are the fixed parameters relating to the coadministration of NEWAEDs, VPA, and EIAEDs, respectively.

Table 2. All model development process and statistical analysis.

$\triangle \mathrm{OBJ}$, the change of objective function value while adding or deleting one covariate from prior model. 
Table 3. Population pharmacokinetic parameters of oxcarbazepine for basic and final models.

\begin{tabular}{|c|c|c|c|c|}
\hline Parameter & Estimate & SE & RSE (\%) & $95 \% \mathrm{Cl}$ \\
\hline \multicolumn{5}{|l|}{ Basic model } \\
\hline$\theta_{K a}\left(h^{-1}\right)$ & 0.46 & 0.0447 & 9.77 & $0.3696-0.5449$ \\
\hline$\theta_{V \mathrm{~d}}(\mathrm{~L} / \mathrm{kg})$ & 0.98 & 0.0376 & 3.82 & $0.9088-1.0562$ \\
\hline$\theta_{\mathrm{CL}}\left(\mathrm{L} \cdot \mathrm{kg}^{-1} \cdot \mathrm{h}^{-1}\right)$ & 0.031 & 0.0013 & 4.18 & $0.0280-0.0331$ \\
\hline$\omega_{\text {ка }}(\%)$ & 55.80 & 24.62 & 44.12 & 7.55-104.06 \\
\hline$\omega_{V d}(\%)$ & 39.98 & 8.15 & 20.38 & $24.01-55.95$ \\
\hline$\omega_{\mathrm{CL}}(\%)$ & 20.24 & 2.79 & 13.78 & $14.78-25.71$ \\
\hline$\sigma(\mathrm{mg} / \mathrm{L})$ & 1.71 & 0.2516 & 14.71 & $1.2168-2.2041$ \\
\hline \multicolumn{5}{|l|}{ Final model } \\
\hline$\theta_{\text {Ka }}\left(\mathrm{h}^{-1}\right)$ & 0.83 & 0.1115 & 13.37 & $0.6152-1.0527$ \\
\hline$\theta_{V \mathrm{~d}}(\mathrm{~L} / \mathrm{kg})$ & 0.67 & 0.0441 & 6.62 & $0.5795-0.7526$ \\
\hline$\theta_{\mathrm{CL}}\left(\mathrm{L} \cdot \mathrm{kg}^{-1} \cdot \mathrm{h}^{-1}\right)$ & 0.035 & 0.0006 & 1.74 & $0.0334-0.0357$ \\
\hline$\theta_{\text {NEWAEDS }}$ & 0.10 & 0.0416 & 39.66 & $0.0233-0.1866$ \\
\hline$\theta_{\mathrm{VPA}}$ & -0.03 & 0.0129 & 42.85 & $-0.0558-0.0049$ \\
\hline$\theta_{\text {EIAEDS }}$ & 0.16 & 0.0658 & 41.19 & $0.0306-0.2888$ \\
\hline$\omega_{\text {Ка }}(\%)$ & 39.82 & 10.44 & 26.22 & $19.36-60.28$ \\
\hline$\omega_{V d}(\%)$ & 19.41 & 2.83 & 14.58 & $13.86-24.95$ \\
\hline$\omega_{\mathrm{CL}}(\%)$ & 6.89 & 1.30 & 18.80 & 4.35-9.43 \\
\hline$\sigma(\mathrm{mg} / \mathrm{L})$ & 0.93 & 0.2279 & 24.37 & $0.4878-1.3821$ \\
\hline
\end{tabular}

SE, standard error; RSE (\%), percentage of relative standard error $(100 \% \times$ SE/EST).

The parameter estimates and $95 \%$ CIs of the basic and final models are shown in Table 3. The final estimated MHD $K_{\mathrm{a}}$ was $0.83 \mathrm{~h}^{-1}$; the $V_{\mathrm{d}}$ was $0.67 \mathrm{~L} / \mathrm{kg}$; the $\mathrm{CL}$ was $0.035 \mathrm{~L} \cdot \mathrm{kg}^{-1} \cdot \mathrm{h}^{-1}$ and the corresponding half-life estimate in these subpopulations was $13.3 \mathrm{~h}$; the peak MHD concentration occurred within $3.6 \mathrm{~h}$ of a single dose. In the final model, the interindividual variability of CL decreased to $6.89 \%$ from $20.24 \%$ in the basic model without adjusting the covariates. The residual coefficient of variation for the final model was $0.93 \mathrm{mg} / \mathrm{L}$, which was an improvement from $1.71 \mathrm{mg} / \mathrm{L}$ for the basic model.

\section{Model evaluation results Internal data evaluation}

The robustness of the final model was shown by the bootstrap results (Table 4). The population estimates for the final model were very similar to the median of the bootstrap replicates (relative error was within $\pm 10 \%$ ) and within the $95 \%$ confidence interval obtained from the bootstrap analysis. The goodness-of-fit plots in Figure 2 show that the model predictions were in reasonable agreement with the observed plasma concentrations. No trend in the conditional weighted residuals versus the population predictions or versus the time-afterdose plots was observed. Most of the conditional weighted residuals were evenly distributed around zero and within an $\mathrm{SD}$ of \pm 2 of the normalized units.

\section{External data evaluation}

When finalizing the final model parameters without reesti-
Table 4. Parameter estimates from the final model and bootstrap validation.

\begin{tabular}{|c|c|c|c|c|}
\hline \multirow{2}{*}{ Parameter } & \multirow{2}{*}{$\begin{array}{l}\text { Final model } \\
\text { estimate }\end{array}$} & \multicolumn{2}{|c|}{ Bootstrap estimate } & \multirow{2}{*}{$\begin{array}{l}\text { Relative } \\
\text { error (\%) }\end{array}$} \\
\hline & & Median & $95 \% \mathrm{Cl}$ & \\
\hline$\theta_{\text {Kа }}\left(\mathrm{h}^{-1}\right)$ & 0.83 & 0.86 & $0.1609-1.2000$ & 3.61 \\
\hline$\theta_{V \mathrm{~d}}(\mathrm{~L} / \mathrm{kg})$ & 0.67 & 0.70 & $0.1993-1.0028$ & 4.48 \\
\hline$\theta_{\mathrm{CL}}\left(\mathrm{L} \cdot \mathrm{kg}^{-1} \cdot \mathrm{h}^{-1}\right)$ & 0.035 & 0.032 & $0.0247-0.0388$ & -8.57 \\
\hline$\theta_{\text {NEWAEDS }}$ & 0.10 & 0.09 & $0.0800-0.1200$ & -10.00 \\
\hline$\theta_{\mathrm{VPA}}$ & -0.03 & -0.03 & $-0.1850-0.0015$ & 0.00 \\
\hline$\theta_{\text {EIAEDS }}$ & 0.16 & 0.17 & $0.0387-0.3382$ & 6.25 \\
\hline$\omega_{\text {ка }}(\%)$ & 39.82 & 42.78 & $2.72-82.84$ & 7.43 \\
\hline$\omega_{V d}(\%)$ & 19.41 & 21.13 & $4.10-38.16$ & 8.86 \\
\hline$\omega_{\mathrm{Cl}}(\%)$ & 6.89 & 7.25 & $0.65-13.84$ & 5.22 \\
\hline$\sigma(\mathrm{mg} / \mathrm{L})$ & 0.93 & 0.92 & $0.3044-1.3807$ & -1.08 \\
\hline
\end{tabular}

Relative error $(\%)=100 \% \times($ Bootstrap median - Final model estimate $) /$ Final model estimate.

mation, 223 MHD concentrations from 115 patients from the validation group were used to validate the final model. These observed concentrations were compared with predicted concentrations calculated from parameters obtained from the modeling group to determine the predictive performance. The MPE, MAE, and MSE are defined in Table 5. All indicators in the final model decreased from the basic model and showed better prediction performance. Scatter plots (Figure 3) were taken to demonstrate the deviations of predictive and observed concentrations pairs. The determination coefficient of the individual value observed/predicted by the final model was 0.9909 . The indicators and plots obtained from final model showed accurate and precise predictions.

Table 5. The prediction accuracy of final model.

\begin{tabular}{lcc}
\hline \multirow{2}{*}{ Error } & \multicolumn{2}{c}{ Mean estimate $(95 \% \mathrm{Cl})$} \\
& Basic model & Final model \\
\hline MPE $(\mathrm{mg} / \mathrm{L})$ & $-0.19 \pm 0.95(-0.32-0.07)$ & $0.01 \pm 0.63(-0.07-0.10)$ \\
MAE $(\mathrm{mg} / \mathrm{L})$ & $0.72 \pm 0.64(0.64-0.81)$ & $0.46 \pm 0.43(0.40-0.51)$ \\
$\mathrm{MSE}(\mathrm{mg} / \mathrm{L})^{2}$ & $0.93 \pm 1.84(0.69-1.17)$ & $0.39 \pm 0.91(0.27-0.51)$ \\
\hline
\end{tabular}

\section{Effects of antiepileptic drugs on MHD}

The typical MHD clearance values estimated in the final model with coadministration of NEWAEDs (LEV, LTG, TPM) and EIAEDs (CBZ, PB) were 10.5\% and 17.4\% higher, respectively, than with no coadministration (Table 3). However, with coadministration of VPA, the clearance of MHD decreased by 3\%. We integrated the data (original plus external) to further verify these effects. Regarding the coadministered AEDs, the combined patient data set ( $n=688$ cases) was divided into 4 groups as follows: (1) OXC monotherapy group [563 cases (81.8\%) with 1078 courses of treatment], (2) OXC+NEWAEDs group [59 cases $(8.6 \%)$ with 72 courses of treatment], (3) OXC+VPA 

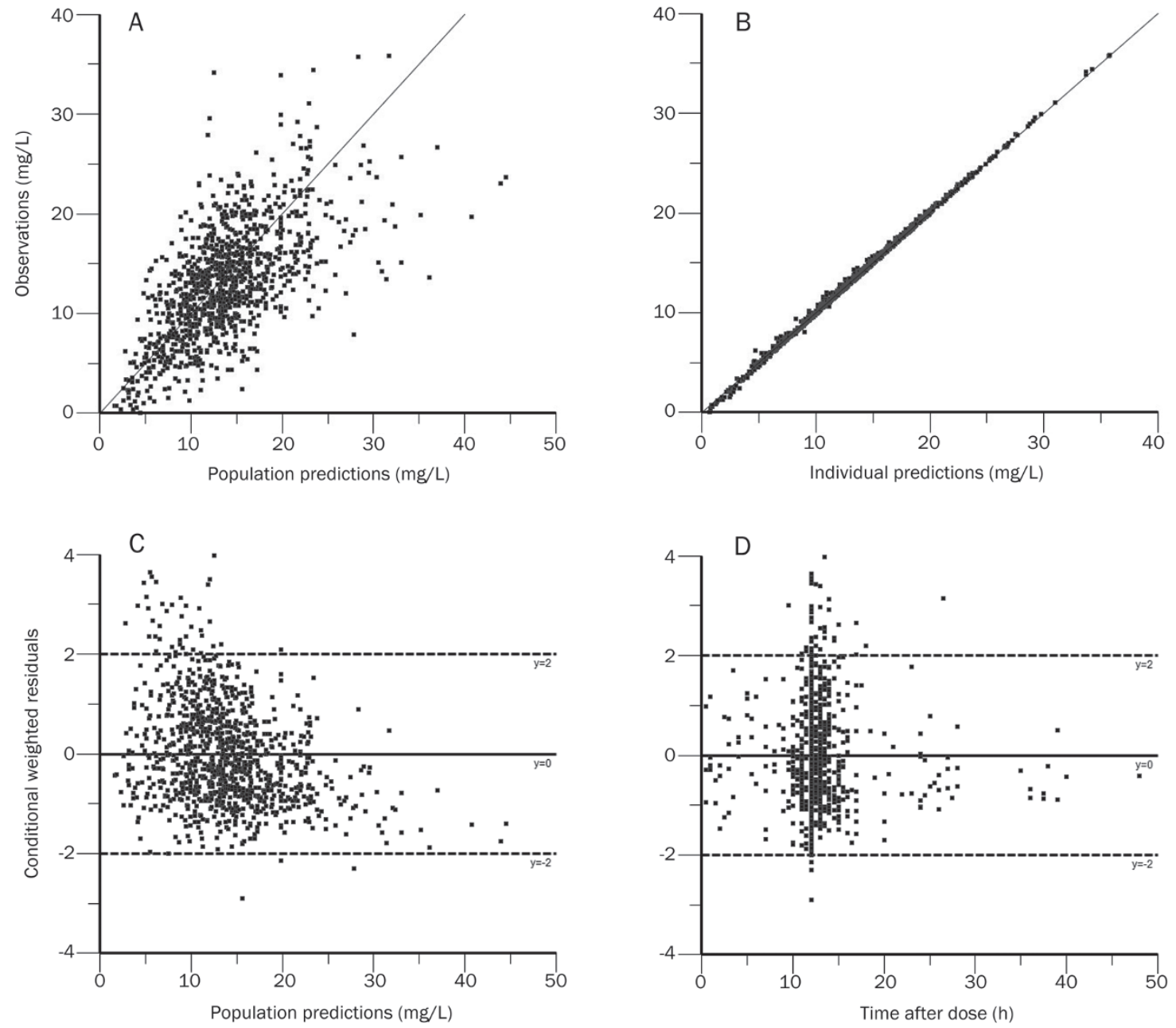

Figure 2. Diagnostic plots of the final PK model in modeling group. Observations against population (A) or individual (B) predictions. The line of identity is shown. Conditional weighted residuals against population predictions (C) or time after dose (D). The line where conditional weighted residuals are equal to 0 is shown.
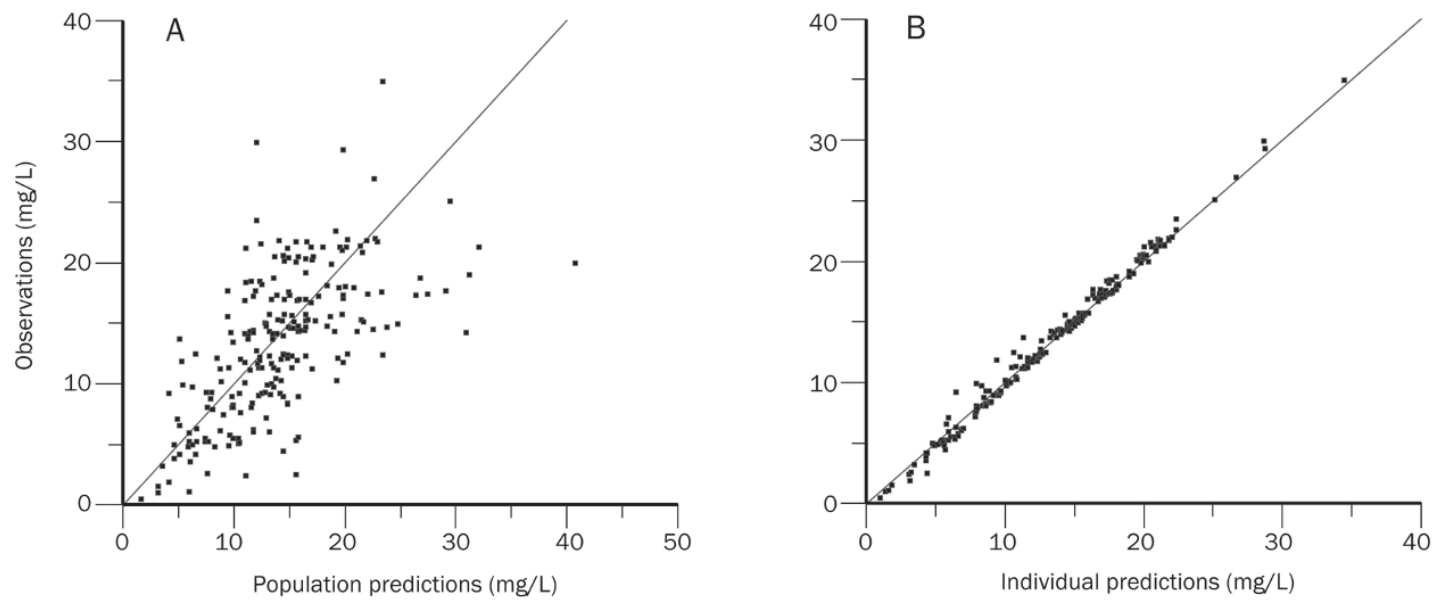

Figure 3. Scatter plots of observations against population (A) or individual (B) predictions in validation group obtained from final model. 
group [42 cases $(6.1 \%)$ with 73 courses of treatment], and (4) OXC+EIAEDs group [24 cases $(3.5 \%)$ with 26 courses of treatment]. The individual apparent clearance of MHD for each course of treatment was estimated with Bayesian and final model parameters. There were significant differences in the MHD clearances between the 4 drug groups $(P<0.01$; Figure 4). The OXC+EIAEDs group had MHD clearances that were higher than the monotherapy group $\left(0.038 \pm 0.009 \mathrm{~L} \cdot \mathrm{kg}^{-1} \cdot \mathrm{h}^{-1}\right.$ vs $\left.0.034 \pm 0.006 \mathrm{~L} \cdot \mathrm{kg}^{-1} \cdot \mathrm{h}^{-1} ; P=0.036\right)$; the OXC+NEWAEDs group also had clearances that were higher than the monotherapy group $\left(0.037 \pm 0.008 \mathrm{~L} \cdot \mathrm{kg}^{-1} \cdot \mathrm{h}^{-1}\right.$ vs $\left.0.034 \pm 0.006 \mathrm{~L}^{\cdot \mathrm{kg}^{-1}} \cdot \mathrm{h}^{-1} ; P=0.001\right)$. In addition, the OXC+VPA group had clearances that were slightly lower than the monotherapy group, but no significant difference $\left(0.033 \pm 0.005 \mathrm{~L} \cdot \mathrm{kg}^{-1} \cdot \mathrm{h}^{-1}\right.$ vs $0.034 \pm 0.006 \mathrm{~L} \cdot \mathrm{kg}^{-1} \cdot \mathrm{h}^{-1}$; $P=0.159)$; the OXC+NEWAEDs group had clearances similar to those of the OXC+EIAEDs group $\left(0.037 \pm 0.008 \mathrm{~L} \cdot \mathrm{kg}^{-1} \cdot \mathrm{h}^{-1}\right.$ vs $\left.0.038 \pm 0.009 \mathrm{~L}^{\mathrm{kg}}{ }^{-1} \cdot \mathrm{h}^{-1} ; P=0.725\right)$.

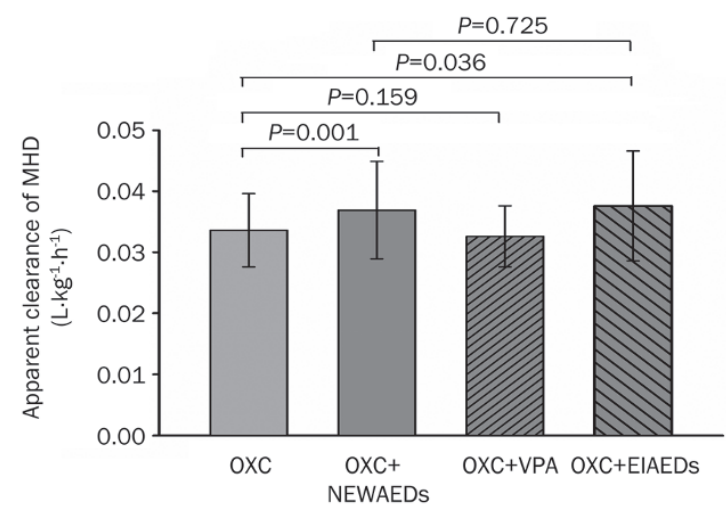

Figure 4. Influence of comedication on MHD apparent clearance: OXC monotherapy group $(n=1078)$; OXC+NEWAEDs group $(n=72)$; OXC+VPA group ( $n=73$ ); OXC+EIAEDs group $(n=26)$.

\section{Discussion}

Although several studies ${ }^{[7,8,17,18]}$ have addressed OXC PK, only a few studies ${ }^{[2,3]}$ using a nonlinear mixed-effect modeling method have been conducted, especially in epileptic children. Consequently, the rationale for the design of the present study was to characterize MHD (the active metabolite of oxcarbazepine) clearance, the covariates affecting its variability, and unexplained residual and interindividual variabilities in Chinese epileptic children. In this study, nonlinear mixedeffect modeling was performed and evaluated based on the extended least square (ELS) principle. The stability and the predictive performance of the final PPK model were established using goodness-of-fit, bootstrapping and external data evaluation.

In the structural model selection step, we observed that a one-compartment model with first-order absorption and elimination best characterized the PK in Chinese epileptic children aged 2 months to 18 years according to a preliminary analysis of the goodness-of-fit. This result is the same as previous modeling performed in other studies ${ }^{[1-3,19]}$. The typical population values of the pharmacokinetic parameters estimated in final model were as follows: $\theta_{K a}=0.83 \mathrm{~h}^{-1}, \theta_{V \mathrm{~d}}=0.67$

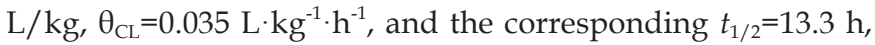
$t_{\max }=3.6 \mathrm{~h}$. These values are similar to the existing parameters that were reported in traditional PK studies ${ }^{[4,5,7,8,18,20]}$ focusing on healthy adult subjects $\left(V_{\mathrm{d}}=0.7-0.8 \mathrm{~L} / \mathrm{kg}\right.$ or $49 \mathrm{~L}, t_{1 / 2}=7-20 \mathrm{~h}$, $\left.t_{\max }=3-12 \mathrm{~h}\right)$. The typical absorption rate constant $\left(\theta_{K \mathrm{Ka}}\right)$ of this study was higher than was found in pediatric patients aged 4 to 17 years in an earlier PPK study $\left(\theta_{K a}=0.598 \mathrm{~h}^{-1}\right)^{[2]}$, but lower than in adults $\left(\theta_{K a}=1.0 \mathrm{~h}^{-1}\right)^{[19]}$. In the current study, the typical apparent volume of distribution $\left(\theta_{V \mathrm{~d}}\right)$ was lower than Northam et $a l^{[1]}$ found in infants and young children aged 1 month to 4 years $\left[\theta_{V \mathrm{~d}}=1.45 \pm 0.11 \mathrm{~L} / \mathrm{kg}\right.$ (monotherapy or concomitant nonenzyme-inducing antiepileptic drugs) or $\theta_{V \mathrm{~d}}=1.39 \pm 0.23 \mathrm{~L} / \mathrm{kg}$ (concomitant enzyme-inducing antiepileptic drugs)], but similar to Park's result ${ }^{[3]}\left(\theta_{V d}=49 \mathrm{~L}\right.$, equals $0.78 \mathrm{~L} / \mathrm{kg}$ normalized by a typical weight of $62.8 \mathrm{~kg}$ ). It appeared that the typical weight-normalized apparent clearance $\left(\theta_{\mathrm{CL}}\right)$ was approximately same in Park's study ${ }^{[3]}\left(\theta_{\mathrm{CL}}=2.13 \mathrm{~L} / \mathrm{h}\right.$, equals 0.034 $\mathrm{L} \cdot \mathrm{kg}^{-1} \cdot \mathrm{h}^{-1}$ normalized by a typical weight of $62.8 \mathrm{~kg}$ ), but lower than that reported in Northam's study ${ }^{[1]}\left[\theta_{\mathrm{CL}}=0.071 \pm 0.015\right.$ $\mathrm{L} \cdot \mathrm{kg}^{-1} \cdot \mathrm{h}^{-1}$ (monotherapy or concomitant non-enzyme-inducing antiepileptic drugs) or $\theta_{\mathrm{CL}}=0.096 \pm 0.024 \mathrm{~L} \cdot \mathrm{kg}^{-1} \cdot \mathrm{h}^{-1}$ (concomitant enzyme-inducing antiepileptic drugs)]. In this study, the age of patients ranged from 2 months to 18 years, of which the patients aged 1 to 14 years accounted for $93.5 \%$. Consequently, the model was more reliable for children aged 1 to 14 years. As the typical population values of the pharmacokinetic parameters show, the pharmacokinetic profile of Chinese children was similar to that of Korean ${ }^{[3]}$ while different from that of westerners ${ }^{[1,2]}$.

It has been suggested in previous studies that factors affecting the pharmacokinetics of MHD might include comedication, age, renal impairment, pregnancy, and compliance ${ }^{[6,21]}$. Our findings suggest that comedication with other AEDs is a significant predictor for MHD clearance in the target population. No association was found between the pharmacokinetics of MHD and the sex, age, weight, or body surface area of the patient.

Because the metabolism of OXC or MHD barely uses catalytic oxidation by CYP450 (only approximately $4 \%$ of MHD is oxidized to the inactive dihydroxy derivative), there is only a small potential for the metabolism of OXC or MHD to be affected by the inducers of CYP ${ }^{[2,5,16]}$. However, some studies suggest that the classical enzyme-inducing AEDs such as CBZ, PB and PHT may increase the metabolism of MHD. Tartara et a ${ }^{[20]}$ reported that the metabolism of OXC $(600 \mathrm{mg}$ single dose) was induced approximately $30 \%$ by $\mathrm{PB}$, as demonstrated by a lower plasma concentration of MHD. McKee et al ${ }^{[22]}$ found that CBZ and PHT decreased the AUC of MHD by $40 \%$ and $29 \%$, respectively. In previous population pharmacokinetic modeling studies ${ }^{[2,3,23]}, \mathrm{CBZ}, \mathrm{PB}$, or PHT administered with OXC increased the apparent clearance of the MHD metabolite by $31 \%$ to $75 \%$. The mechanism of this heteroinduction is unclear. However, enzyme-inducing AEDs might enhance the metabolism of MHD through the induction of 
UGT or CYP enzymes ${ }^{[11]}$, and the former pathway may play a more important role. Our study identified a slighter inducing effect of enzyme inducers on OXC metabolism than was reported in the above studies. The final model indicated that enzyme-inducing AEDs, such as CBZ and PB, administered with OXC increased the weight-normalized apparent clearance of MHD only by $17.4 \%$. This inducing effect was less pronounced but also significant. It also explains why some studies ${ }^{[10]}$ failed to show a significant effect for enzyme-inducing AEDs on the MHD concentration.

The majority of the newer generation AEDs are usually regarded as free of enzyme-inducing properties and therefore unlikely to interact with OXC. However, there are limited data available on this inference. In the current study, we showed that comedication of some newer generation AEDs such as LEV, LTG, and TPM with OXC also could increase the weight-normalized apparent clearance of MHD by 10.5\%. LEV is neither bound to serum proteins, nor metabolized by CYP450 oxidative processes following absorption. Its major route of elimination is renal, with approximately $66 \%$ of a dose eliminated unchanged and $27 \%$ as inactive metabolites. The major metabolic route is hydrolysis in the blood and various tissues to LO57 and other minor inactive metabolites ${ }^{[6]}$, thus it is unlikely to affect the metabolism of MHD for the above reasons. The majority of LTG is conjugated with glucuronic acid and subsequently excreted in the urine without CYPmediated metabolism. In the same metabolic pathway, OXC is also primarily cleared by glucuronic acid conjugation ${ }^{[24]}$. For this reason, potential interactions may occur in patients receiving combination therapy of LTG and OXC. Some studies $^{[13,14]}$ reported that OXC might induce the UGT1A4-mediated metabolism of LTG, as demonstrated by a $29 \%$ decrease in its serum concentration. However, the influence of LTG on the metabolism of OXC is rarely reported, and there is a lack of evidence supporting it. Sallas $e a^{[2]}$ found a trend toward patients with lower LTG concentrations having higher MHD concentrations. A significant increase in the clearance of both LTG and MHD occurred in two pregnant women who received combination therapy of LTG and OXC in Wegner's case report ${ }^{[24]}$. The above observations and our findings suggest that LTG might have catalytic effects on the UGTmediated metabolism of MHD. The isoforms of UGT involved in MHD glucuronidation have not yet been identified ${ }^{[24]}$, thus this hypothesis requires further validation. Some studies ${ }^{[14,25]}$ showed a modest inducing effect of higher doses (up to 200 $\mathrm{mg} / \mathrm{d}$ ) of TPM on CYP3A4 and possible effects on the activity of UGTs. Thus, the CYP and UGT-mediated metabolism of MHD might also be induced by a high dose of TPM. To test the above analysis, the patients in the OXC+NEWAEDs group were divided into OXC+LEV, OXC+LTG, and OXC+TPM subgroups. The result indicated that the OXC+LEV group had similar clearances to that of the OXC monotherapy group $\left(0.034 \pm 0.008 \mathrm{~L} \cdot \mathrm{kg}^{-1} \cdot \mathrm{h}^{-1}\right.$ vs $\left.0.034 \pm 0.006 \mathrm{~L} \cdot \mathrm{kg}^{-1} \cdot \mathrm{h}^{-1} ; P=0.979\right)$, while the OXC+LTG group and the OXC+TPM group had higher MHD clearances than the monotherapy group $(0.038 \pm 0.008$ $\mathrm{L} \cdot \mathrm{kg}^{-1} \cdot \mathrm{h}^{-1}$ and $0.039 \pm 0.004 \mathrm{~L} \cdot \mathrm{kg}^{-1} \cdot \mathrm{h}^{-1}$ vs $0.034 \pm 0.006 \mathrm{~L} \cdot \mathrm{kg}^{-1} \cdot \mathrm{h}^{-1}$,
$P<0.05)$. In conclusion, the increase in MHD clearance in our study might be caused by the inducing effect of coadministered LTG or TPM. We also found that VPA comedication with OXC decreased the clearance of MHD by 3\%, but it failed to show a significant effect on the study of integrated data (original plus external) (Figure 4). As an inhibitor of UGT enzymes, VPA has been shown to have a potent inhibiting effect on the glucuronidation of $\mathrm{LTG}^{[2]}$. Although OXC is also primarily cleared by glucuronic acid conjugation, the majority of studies ${ }^{[20,22]}$ found there were no drug-drug interactions for VPA used with OXC. Tartara et al ${ }^{[20]}$ suggested that the glucuronyl transferase isozymes catalyzing MHD conjugation differ from those responsible for the glucuronidation of LTG. Our study showed a slight inhibiting effect of VPA on MHD metabolism, but it is not statistically and clinically significant.

Some studies ${ }^{[2,3,11,26]}$ suggested that the weight-normalized value of MHD clearance decreased as the patient age increased. Compared with adults, the average magnitude of the increase in MHD clearance values has been found to be on the order of $30 \%-160 \%$ in children ${ }^{[26]}$. A significant and positive correlation between the MHD concentration-to-OXC dose ratio (CDR) and patient age was found in Armijo's study ${ }^{[11]}$. As a result, children require larger dosages (milligrams per kilogram) to achieve serum drug levels comparable with those found in adults ${ }^{[6]}$. However, many PPK studies ${ }^{[2,3]}$ failed to find age but body size (such as weight or body surface area) was included in the final model as a statistically meaningful covariate for the clearance of MHD. Furthermore, once the best relationship for clearance in terms of body size was determined, one often found that there was no difference in the clearances between children and adults adjusted for body size $^{[2]}$. In the current study, patient body weight was included into the full regressive model as a statistically meaningful covariate for CL (Table 2), but it was eliminated from the model in the covariates backward analysis step using a more stringent criterion of statistical significance $\left(X^{2}=6.63, P<0.01\right)$.

A study ${ }^{[27]}$ suggested that the clearance of OXC and MHD is reduced in patients with impaired renal function (creatinine clearance $<30 \mathrm{~mL} / \mathrm{min}$ ) and that the elimination half-life of MHD is prolonged with a corresponding 2-fold increase in AUC. A limitation of the current study is that the patient renal functions have not been incorporated into the covariate analyses, thus the conclusion is only applicable to children with normal liver and kidney function during OXC treatment. Future studies will investigate the OXC pharmacokinetic characteristics in populations with special pathological conditions, such as liver and kidney dysfunction.

Our findings indicated that enzyme-inducing AEDs and some newer AEDs (including LTG and high dose TPM) could induce the metabolism of MHD, and hence decrease the serum drug concentration below the therapeutic window, which may lead to epileptic seizure. Thus, we suggest that MHD concentrations should be monitored when OXC is coadministered with the above enzyme inducers, and the dosage of OXC should be adjusted accordingly. The PPK model established in this study will facilitate individualized dosage regimens for 
OXC.

\section{Conclusion}

The population analysis was successful in describing the pharmacokinetics of OXC in Chinese children aged 2 months to 18 years. A one-compartment pharmacokinetic model with first-order absorption adequately described the data, while the exponential model was used to describe the inter-individual variability and the addition model described the intra-individual variability. The final PPK model was shown to be stable and effective in the prediction of serum MHD concentrations by internal and external validation. The findings indicate that the apparent clearance of MHD increased slightly with comedication with enzyme-inducing AEDs and some newer generation AEDs. This study will facilitate individualized dosage regimens.

\section{Acknowledgements}

This project was supported by the Scientific Research Foundation of Clinical Medicine of Wuhan City (№ WX14C47).

\section{Author contribution}

Yang WANG and Hua-nian ZHANG designed research; Yu-jun CHEN, Jing PENG, and Mao-chang LIU performed research; Hua-nian ZHANG and Hua XU contributed new analytical tools and reagents; Yang WANG analyzed data; Yang WANG, Chang-he NIU, and Ping GAO wrote the paper.

\section{References}

1 Northam RS, Hernandez AW, Litzinger MJ, Minecan DN, Glauser TA, Mangat $\mathrm{S}$, et al. Oxcarbazepine in infants and young children with partial seizures. Pediatr Neurol 2005; 33: 337-44.

2 Sallas WM, Milosavljev S, D'Souza J, Hossain M. Pharmacokinetic drug interactions in children taking oxcarbazepine. Clin Pharmacol Ther 2003; 74: 138-49.

3 Park KJ, Kim JR, Joo EY, Seo DW, Hong SB, Ko JW, et al. Drug interaction and pharmacokinetic modeling of oxcarbazepine in korean patients with epilepsy. Clin Neuropharmacol 2012; 35: 40-4.

4 May DTW, Korn-Merker E, Rambeck B. Clinical pharmacokinetics of oxcarbazepine. Clin Pharmacokinet 2003; 42: 1023-42.

5 Flesch G. Overview of the clinical pharmacokinetics of oxcarbazepine. Clin Drug Investig 2004; 24: 185-203.

6 Patsalos PN, Berry DJ, Bourgeois BF, Cloyd JC, Glauser TA, Johannessen $\mathrm{SI}$, et al. Antiepileptic drugs-best practice guidelines for therapeutic drug monitoring: a position paper by the subcommission on therapeutic drug monitoring, ILAE Commission on Therapeutic Strategies. Epilepsia 2008; 49: 1239-76.

7 Volosov A, Xiaodong S, Perucca E, Yagen B, Sintov A, Bialer M. Enantioselective pharmacokinetics of 10-hydroxycarbazepine after oral administration of oxcarbazepine to healthy Chinese subjects. Clin Pharmacol Ther 1999; 66: 547-53.

8 Flesch G, Czendlik C, Renard D, Lloyd P. Pharmacokinetics of the monohydroxy derivative of oxcarbazepine and its enantiomers after a single intravenous dose given as racemate compared with a single oral dose of oxcarbazepine. Drug Metab Dispos 2011; 39: 1103-10.

9 Schmutz M, Brugger F, Gentsch C, McLean MJ, Olpe HR. Oxcarbazepine: preclinical anticonvulsant profile and putative mechanisms of action. Epilepsia 1994; 35 Suppl 5: S47-50.
10 Viola MS, Bercellini MA, Saidón P, Rubio MC. Pharmacokinetic variability of oxcarbazepine in epileptic patients. Medicina 2000; 60: 914-8

11 Armijo JA, Vega-Gil N, Shushtarian M, Adin J, Herranz JL. 10-Hydroxycarbazepine serum concentration-to-oxcarbazepine dose ratio: influence of age and concomitant antiepileptic drugs. Ther Drug Monit 2005; 27: 199-204.

12 Hossain M, Sallas W, Gasparini M, D Souza J. Drug-drug interaction profile of oxcarbazepine in children and adults. Neurology 1999; 52: 525.

13 May TW, Rambeck B, Jurgens U. Influence of oxcarbazepine and methsuximide on lamotrigine concentrations in epileptic patients with and without valproic acid comedication: results of a retrospective study. Ther Drug Monit 1999; 21: 175-81.

14 Johannessen SI, Landmark CJ. Antiepileptic drug interactions principles and clinical implications. Curr Neuropharmacol 2010; 8: 254-67.

15 Lloyd P, Flesch G, Dieterle W. Clinical pharmacology and pharmcokinetics of oxcarbazepine. Epilepsia 1994; 35: 10-3.

16 Schutz H, Feldmann KF, Faigle JW, Kriemler HP, Winkler T. The metabolism of ${ }^{14} \mathrm{C}$-oxcarbazepine in man. Xenobiotica 1986; 16 : 769-78.

17 Kim DW, Gu N, Jang IJ, Chu K, Yu KS, Cho JY, et al. Efficacy, tolerability, and pharmacokinetics of oxcarbazepine oral loading in patients with epilepsy. Epilepsia 2012; 53: e9-12.

18 Flesch G, Tudor D, Souppart C, D'Souza J, Hossain M. Oxcarbazepine final market image tablet formulation bioequivalence study after single administration and at steady state in healthy subjects. Int J Clin Pharmacol Ther 2002; 40: 524-32.

19 Wegner I, Wilhelm AJ, Sander JW, Lindhout D. The impact of age on lamotrigine and oxcarbazepine kinetics: a historical cohort study. Epilepsy Behav 2013; 29: 217-21.

20 Tartara A, Galimberti CA, Manni R, Morini R, Limido G, Gatti G, et al. The pharmacokinetics of oxcarbazepine and its active metabolite 10-hydroxy-carbazepine in healthy subjects and in epileptic patients treated with phenobarbitone or valproic acid. $\mathrm{Br} J$ Clin Pharmacol 1993; 36: 366-8.

21 Bring $\mathrm{P}$, Ensom $\mathrm{MH}$. Does oxcarbazepine warrant therapeutic drug monitoring? A critical review. Clin Pharmacokinet 2008; 47: 767-78.

22 McKee PJ, Blacklaw J, Forrest G, Gillham RA, Walker SM, Connelly D, et al. A double-blind, placebo-controlled interaction study between oxcarbazepine and carbamazepine, sodium valproate and phenytoin in epileptic patients. Br J Clin Pharmacol 1994; 37: 27-32.

23 Johannessen LC, Baftiu A, Tysse I, Valso B, Larsson PG, Rytter E, et al. Pharmacokinetic variability of four newer antiepileptic drugs, lamotrigine, levetiracetam, oxcarbazepine, and topiramate: a comparison of the impact of age and comedication. Ther Drug Monit 2012; 34: 440-5.

24 Wegner I, Edelbroek P, de Haan GJ, Lindhout D, Sander JW. Drug monitoring of lamotrigine and oxcarbazepine combination during pregnancy. Epilepsia 2010; 51: 2500-2.

25 Nallani SC, Glauser TA, Hariparsad N, Setchell K, Buckley DJ, Buckley AR, et al. Dose-dependent induction of cytochrome P450 (CYP) 3A4 and activation of pregnane $X$ receptor by topiramate. Epilepsia 2003; 44: 1521-28.

26 Perucca E. Pharmacokinetic variability of new antiepileptic drugs at different ages. Ther Drug Monit 2005; 27: 714-7.

27 Rouan MC, Lecaillon JB, Godbillon J, Menard F, Darragon T, Meyer $\mathrm{P}$, et al. The effect of renal impairment on the pharmacokinetics of oxcarbazepine and its metabolites. Eur J Clin Pharmacol 1994; 47: 161-7. 